\title{
LA LABOR editorial de LeSter EMbree SOBRe Alfred SchutZ
}

\section{THE EDITORIAL WORK OF LeSTer EMBREe ON ALfRED SCHUTZ}

\author{
Jacobo López Villalba \\ Conservatorio Profesional de Música de la Comunidad de Madrid / Doctorando \\ UNED jacobolvillalba@hotmail.com
}

\begin{abstract}
Resumen: El presente artículo tiene por objeto la elaboración de una bibliografía que ilustre la tarea editorial que realizó Lester Embree sobre la figura y el pensamiento de Alfred Schutz. Dicha bibliografía abarca las ediciones sobre textos de Schutz que preparó Embree, algunos inéditos hasta entonces, así como la totalidad de obras que escribió, editó y co-editó sobre Schutz y sus aportaciones en forma de capítulos de libros y artículos de revista a la investigación relacionada con el pensamiento de Alfred Schutz. Acompaña al repertorio bibliográfico un breve comentario acerca de algunas de las principales obras de Embree recogidas en el mismo que nos permite hacer un recorrido por las cuestiones relativas al pensamiento de Schutz que más le interesaron al fenomenólogo americano. También constataremos el interés predominante de Embree por las ciencias culturales y su esfuerzo en promover una mayor colaboración entre fenomenólogos y científicos sociales de diferentes disciplinas.
\end{abstract}

Palabras clave: Lester Embree. Alfred Schutz. Bibliografía. Ciencias sociales. Ciencias culturales. Interdisciplinariedad.

\begin{abstract}
This article aims to present a bibliography that illustrates the editorial work that Lester Embree wrote about Alfred Schutz and his thought. This bibliography covers the editions of Schutz's texts that Embree prepared, some unpublished until then, as well as all the works that he wrote, edited and co-edited about Schutz and his contributions to the research of Alfred Schutz's thought in form of chapters of collective works and papers in Journals. We include a brief comment about some of the main works of Embree taken from the bibliography which allows us to explore with the american phenomenologist some questions regarding Schutz's thought. We will also take note of Embree's interest in cultural sciences and his endeavours to promote a deeper collaboration between phenomenologists and social scientists from different disciplines.
\end{abstract}

Keywords: Lester Embree. Alfred Schutz. Schutzian bibliography. Social sciences. Cultural sciences. Interdisciplinarity.

\section{INTRODUCCIÓN}

El presente artículo tiene por objeto la elaboración de una bibliografía que ilustre la tarea editorial que realizó Lester Embree sobre la figura y el pensamiento de Alfred Schutz. Para ello el autor se ha basado entre otras fuentes 
en el Curriculum Vitae que el propio Embree publicó en $2010^{1}$, realizando una búsqueda bibliográfica desde dicho año hasta la actualidad. El repertorio bibliográfico que supone el cuerpo principal del presente artículo se ha confeccionado según diferentes criterios. Por una parte se han incluido los textos de Alfred Schutz que Embree publicó, ya fuesen anteriormente inéditos o reediciones que el pensador norteamericano incluyó en obras colectivas. Dicha labor de edición de textos de Schutz comenzó en 1972 con un trabajo de 1945 recogido en un volumen dedicado a Aron Gurwitsch y culminó en 2011 con la edición del importante volumen cinco de Collected Papers. Las referencias bibliográficas se han ordenado por fecha de escritura, que suele coincidir con la primera publicación de Schutz en revista, pero no con la de los Collected Papers, volúmenes al que se suelen referir los fragmentos citados en casi todos los estudios sobre Schutz. Es de vital importancia conocer e incluir la fecha de escritura de los textos de Schutz, puesto que a excepción de Der sinnhafte Aufbau der sozialen Welt de $1932^{2}$, todos los volúmenes de su obra que recogen diferentes trabajos se editaron después de su muerte. En el caso de que los textos de Schutz editados por Embree aparezcan posteriormente en otra publicación suya, se ha incluído también esa referencia. Si los textos aparecieron antes en alguna otra publicación se ha indicado únicamente el volumen de Collected Papers al que pertenecen. No debemos perder de vista que la bibliografía recogida está centrada en la obra de Embree sobre Schutz y con este criterio se ha confeccionado también la primera sección de la misma.

El segundo apartado de la bibliografía está dedicado a libros que Embree escribió, editó o co-editó. La mayor parte de ellos pertenece a la serie Contributions to Phenomenology que, en colaboración con el Center for Advanced Research in Phenomenology (CARP), primero Kluwer Academic Publishers y posteriormente Springer editaron desde 1987. En el caso de que en la obra colectiva co-editada no haya ninguna contribución de Embree sobre Schutz pero sí de otro autor, se ha incluido el título y el nombre del autor entre paréntesis. Es importante resaltar que el autor del presente artículo no se ha limitado a buscar las referencias que contuviesen la palabra "Schutz" en el título o en el

1 Lester Embree, "Curriculum Vitae", en Thomas Nenon / Philip Blosser (eds.), Advancing Phenomenology: Essays in Honor of Lester Embree, Dordrecht: Springer, 2010, pp. 471-509.

${ }^{2}$ Alfred Schutz, Der sinnhafte Aufbau der sozialen Welt: Eine Einleitung in die verstehende Soziologie, Viena: Springer, 1932. 
resumen. Se ha tratado de realizar una bibliografía lo más exhaustiva posible y ello incluye rastrear todas las alusiones de Embree a la figura de Schutz, de manera que si una vez estudiado el texto el autor considera que dichas alusiones son significativas se ha añadido al presente repertorio. Esto quiere decir que el autor ha tenido acceso al contenido completo de casi la totalidad de las referencias bibliográficas que forman el cuerpo del presente artículo.

La tercera sección del repertorio bibliográfico está dedicada a capítulos de libros y artículos de revista. En esta sección aparecen aportaciones de Embree sobre Schutz que pertenecen tanto a libros editados por él mismo junto con otros autores como a aportaciones que el fenomenólogo americano hizo para otros volúmenes colectivos, especialmente en la mencionada serie Contributions to Phenomenology. También se recogen aquí los numerosos artículos de revista centrados en Schutz o que contienen alusiones suficientes para incluirse en el repertorio que nos ocupa. Algunos artículos aparecieron por primera vez en traducciones a otros idiomas y otros muchos se han traducido después. Se ha hecho la referencia oportuna en el caso de que se trate de lenguas oficiales del Estado español.

Aunque el presente artículo no pretende ni puede ser por extensión un análisis de la influencia del pensamiento de Alfred Schutz en la obra de Lester Embree, se incluyen unas consideraciones generales al hilo de varias obras que el autor valora como especialmente relevantes en el recorrido del fenomenólogo americano sobre Schutz. De la misma manera, el autor considera que el estudio atento de dichas obras que se comentan brevemente a continuación sería de vital importancia para cualquier estudioso de la filosofía de Alfred Schutz. En el momento de la elaboración del presente artículo, tengo noticias de un homenaje que la Society for Phenomenology and the Human Sciences (SPHS), en colaboración con la Society for Phenomenology and Existential Philosophy (SPEP) dedica a Lester Embree en la Universidad de Memphis en el marco de su Conferencia Anual, los días 19-21 de Octubre de 2017. En dicha cita el profesor Andreas Göttlich, de la Universidad de Konstanz, se encargará de la conferencia "Passing on the Baton: Lester Embree's Involvement with the Work of Alfred 
Schutz", primer trabajo del que tengo constancia sobre la influencia de Alfred Schutz en el pensamiento de Lester Embree ${ }^{3}$.

\section{Comentario a LA LABOR EDITORIAL DE EMBREE SOBRE SCHUTZ: PRINCIPALES HITOS}

El propio Embree nos cuenta cómo su primer contacto con el pensamiento de Schutz tuvo lugar a través de Aron Gurwitsch y cómo se quedó impresionado al estudiar en el verano de 1963 el volumen publicado en 1962 de Collected Papers. El curso siguiente acudió en la New School of Social Research de Nueva York a las enseñanzas de Gurwitsch y Luckmann sobre Schutz. Posteriormente Gurwitsch recomendó a Embree a Ilse Schutz para la confección del índice de Collected Papers, Vol. III (1966) ${ }^{4}$. Embree también realizó el índice de Collected Papers, Vol. IV (1996), aunque no figura en los créditos, la edición es de Helmut Wagner y Fred Kersten, y por supuesto el de Collected Papers, Vol. V (2011). La edición del penúltimo volumen de esta serie hasta la fecha supone la culminación de la tarea de edición de Embree sobre los textos de Alfred Schutz. En este volumen se incluyen algunos textos previamente publicados y en especial uno muy importante en la trayectoria de Schutz publicado en 1970 y en su momento agotado. Se trata del texto Reflections on the Problem of Relevance (1947, 1951). Es destacable la presencia de una selección de la correspondencia que mantuvo Schutz con Parsons, Kaufmann, Voegelin y Gurwitsch, anteriormente publicada en volúmenes separados. Por último, es importante mencionar el texto sobre la Teoría de la Cultura de T.S. Eliot (1953), de especial interés para Embree en relación a su definición de las denominadas Schutzian Cultural Sciences.

Los trabajos recogidos en el volumen publicado en 1988 Worldly Phenomenology: The Continuing Influence of Alfred Schutz on North American Human Science provienen algunos de ellos de unas conferencias pronunciadas en Ohio en junio de 1986. Se trata de unos trabajos centrados en aportaciones de estudiosos de Norteamérica. Embree nos cuenta en la Introducción cómo dicho volumen fue la consecuencia de su interés en la filosofía fenomenológica

\footnotetext{
3 Para una visión general de la contribución de Embree a la fenomenología cfr. Thomas Nenon, "Advancing Phenomenology as a Practical Endeavor", en Thomas Nenon / Philip Blosser, op. cit., pp. 457463. Cfr. también Hisashi Nasu, "Lester E. Embree (January 9, 1938-January 19, 2017)", Human Studies, vol. 40, 1 (2017) 1-6.

${ }^{4}$ Lester Embree, "Its about time! A Sometimes Personal Narrative of Schutz Scholarship", Schutzian Research, vol. 4 (2012), p. 10.
} 
de las ciencias humanas en general y en la obra de Alfred Schutz en particular ${ }^{5}$. Es sabido que para Husserl las ciencias humanas y naturales se consideraban como pertenecientes a la actitud natural y en ese sentido de pertenecer a un mundo que engloba algo más que el mundo natural, Husserl denominaba a las disciplinas de la actitud natural como "mundanas". Embree consideró más oportuno el término "worldly" en lugar de "mundane" y de ahí el título del volumen ${ }^{6}$. Alfred Schutz se adhirió al proyecto de fundar las ciencias sociales e históricas en una psicología de la conciencia humana que se desarrollara a través de la reflexión acerca de temas como el conocimiento de uno mismo, el conocimiento de otros, el pensamiento científico, así como el pensamiento de la vida cotidiana, o la acción práctica7. En este sentido, para Embree el intento de fundamentar las ciencias humanas sociales e históricas en una psicología científica humana es una tarea de la filosofía de la ciencia de igual forma que la ciencia física fundamenta la biología o la química ${ }^{8}$. Observamos que en este primer volumen importante que Embree dedicó al pensamiento de Schutz se perfila la figura del pensador austríaco como un filósofo de las ciencias humanas. A lo largo de los años Embree llegó a la conclusión de que el núcleo del pensamiento schutziano es una teoría de las ciencias culturales ${ }^{9}$. Schutz utiliza los términos Geisteswissenschaft y Kulturwissenschaft, traducidos al inglés por Embree como "human sciences" y "cultural sciencies". Las denominadas Geisteswissenschaften son más extensivas para Schutz que las ciencias sociales (social sciences) en Estados Unidos, ya que Schutz engloba las ciencias históricas, arqueología incluida, considerando la historia como una disciplina de las humanidades ${ }^{10}$. Según Embree, si queremos incluir el estudio de disciplinas que se encarguen de los denominados animales no humanos, cosa que Schutz no llevó a cabo, es más recomendable utilizar la expresión ciencias culturales (cultural sciencies) en lugar de ciencias humanas (human sciences) ${ }^{11}$. Embree nos recuerda que el pensamiento de Schutz, si bien principalmente dirigido a la

\footnotetext{
${ }^{5}$ Lester Embree (ed.), Worldly Phenomenology: The Continuing Influence of Alfred Schutz on North American Human Science, Washington, D.C.: Center for Advanced Research in Phenomenology / University Press of America, 1988, p. XI.

${ }^{6}$ Ibid. p. XII.

${ }^{7}$ Idem.

${ }^{8}$ Idem.

9 Lester Embree, "Cultural Science in Literary Light", en Michael Barber / Jochen Dreher (eds.), The Interrelation of Phenomenology, Social Sciences and the Arts, Dordrecht: Springer, 2014, p. 21.

10 Lester Embree, "Introduction", en Alfred Schutz, Collected Papers. Volumen V: Phenomenology and the Social Sciences, Editado por Lester Embree, Dordrecht: Springer, 2011, p. XII.

${ }^{11}$ Ibid., p. XIII.
} 
fundamentación fenomenólogica de la sociología interpretativa, ha sido también muy apreciado por un amplio espectro de disciplinas científicas humanas. El principal objetivo de la publicación de este volumen es "evaluar el impacto y potencial de Schutz más allá de la sociología y de la filosofía"12. El volumen contiene trece ensayos y una muy interesante bibliografía recopilada por Thomas B. Wall consistente en literatura en inglés sobre Alfred Schutz desde 1933 hasta 1987. Los temas tratados por los diferentes participantes en el volumen incluyen sociología de la ciencia, psicología social, computerización, política, psiquiatría, estudios clínicos, comunicología, dando cuenta de la multidisciplinariedad dentro de la fenomenología que tantas veces Embree estudió y promovió ${ }^{13}$. Embree se refiere a su llegada a Florida Atlantic University en 1990 como un punto de inflexión en su carrera en relación a que comenzó a estudiar a Schutz más profundamente dirigiéndose hacia el estudio de la cultura. La propuesta en 1992 para la publicación de la Encyclopedia of Phenomenology (1997) supuso para Embree el comienzo de una toma de conciencia de lo mucho que la tradición fenomenológica se había extendido por el mundo, así como de su influencia en numerosas disciplinas no filosóficas ${ }^{14}$.

Lester Embree realizó también una valiosa indagación en lo que podría denominarse una Sociología de la Literatura en el pensamiento de Schutz. El fruto de esta construcción realizada a partir de fragmentos diseminados sobre teoría literaria en la obra de Schutz se publicó en 1998 en el volumen Alfred Schutz's "Sociological Aspect of Literature": Construction and Complementary Essays, junto con diez ensayos de algunos de los más prestigiosos fenomenólogos y estudiosos de Schutz como Fred Kersten, Ilja Srubar, Martin Endress, Hisashi Nasu, George Psathas y Michael Barber, entre otros. El origen de este volumen es una página de notas que Schutz utilizó en una conferencia que ofreció el 5 de febrero de 1955 en la New School for Social Research. A partir de esas notas, utilizadas a modo de "boceto arquitectónico", Embree procede a la construcción de un texto utilizando los pasajes dedicados a la literatura

\footnotetext{
12 Lester Embree (ed.), Worldly Phenomenology: The Continuing Influence of Alfred Schutz on North American Human Science, Washington, D.C.: Center for Advanced Research in Phenomenology / University Press of America, 1988, p. XVI.

${ }^{13}$ Cfr. Lester Embree, "Interdisciplinarity within Phenomenology", Indo-Pacific Journal of Phenomenology, vol. 10, 1 (2010) 1-7. Publicado en castellano: "La interdisciplinariedad dentro de la fenomenología", Investigaciones Fenomenológicas 8 (2011) 9-21.

${ }_{14}$ Lester Embree, "Interdisciplinarity within Phenomenology", p. 4; "La interdisciplinariedad dentro de la fenomenología", p. 17.
} 
diseminados en la obra de Schutz. Embree define esta construcción como un "nuevo edificio realizado a partir de copias de otras piezas relevantes de edificios del arquitecto utilizando un diseño del que sólo se conserva un boceto"15. Los intereses principales de Schutz se centraron, además de en la filosofía y la sociología, en la música y en la literatura ${ }^{16}$. Es por esto que la mención de esta obra editada por Embree es de gran importancia en el presente artículo. Quizás se echa de menos una atención mayor por parte de Embree al estudio de la música en Schutz. Sin embargo, este volumen editado por el fenomenólogo americano es de obligada consulta para cualquier estudioso de la fenomenología de la música de Schutz. El boceto que sirve de modelo para la construcción del texto consiste en una matriz de filas y columnas, a través de la cual Schutz confronta la Poesía, el Drama y la Novela atendiendo a diferentes criterios. Estos elementos de estudio se presentan bajo los siguientes términos: lenguaje, situación, relevancia, realidad, motivos, dimensión temporal, relación del autor con su obra y relación de la obra literaria con el oyente. Embree recorre la obra de Schutz en busca de párrafos dedicados a la literatura y los sitúa en cada una de las casillas de esta matriz.

La obra Schutzian Social Science editada por Lester Embree en 1999 tiene su origen en un simposio patrocinado por CARP y Florida Atlantic University que tuvo lugar en 1997. Embree considera dicha obra como una puesta al día de las investigaciones sobre la obra de Schutz e inspiradas en su pensamiento después de una década de la aparición del volumen Worldly Phenomenology: The Continuing Influence of Alfred Schutz on North American Human Science, ya mencionado, y la obra editada por Ilja Srubar y Elizabeth List Alfred Schütz, Neue Beiträge zur Rezeption seines Werkes, ambas de 1988. A diferencia de la obra editada por Embree en 1988, Schutzian Social Science abarca un espectro más internacional, incluyendo aportaciones de Asia y de Europa. En el momento de edición de dicha obra (1999), Embree nos emplazaba a realizar un nuevo seguimiento del estado de la investigación sobre Schutz en otros diez años ${ }^{17}$. Esta puesta al día del estado de la cuestión se realizaría en 2009 en el volumen

\footnotetext{
15 Lester Embree, "A Construction of Alfred Schutz's 'Sociological Aspect of Literature'", en Lester Embree (ed.), Alfred Schutz's "Sociological Aspect of Literature": Construction and Complementary Essays, Dordrecht: Kluwer Academic Publishers, 1998, p. 3

${ }^{16}$ Entrevista a Ilse Schutz, noviembre de 1981, citada en ibid. p. 8.

17 Lester Embree (ed.), Schutzian Social Science, Dordrecht: Kluwer Academic Publishers, 1999, p. XIV.
} 
editado por Nasu, Embree, Psathas y Srubar titulado Alfred Schutz and his Intellectual Partners, que por razones de espacio no podremos comentar. Schutzian Social Science contiene doce ensayos de diferentes autores, incluyendo al propio Embree, que tratan principalmente de política, ciencia social, economía y algunos conceptos centrales en el pensamiento de Schutz como realidades múltiples, tipos ideales y acción humana. Es de destacar el hecho de que, además de ensayos sobre el pensamiento de Schutz, se incluyen estudios que tratan de continuar la labor de Schutz en determinados ámbitos, practicando "Schutzian Social Science", como reza el título de la obra. Este tipo de investigación, en el espíritu de Schutz, sobre temas que él no trató específicamente, se convirtió desde hace unos años en uno de los principales objetivos de Lester Embree, centrando su trabajo sobre Schutz en un estudio de las ciencias culturales con las herramientas del pensador austríaco. Al fin y al cabo una de las recomendaciones del propio Schutz era que los "filósofos se debían dirigir hacia los científicos sociales para aprender de ellos"18. Es sabido cómo Embree animó a los profesionales de diferentes disciplinas culturales a realizar investigaciones fenomenólogicas, y cómo promulgó una mayor colaboración entre fenomenólogos de formación filosófica y científicos culturales ${ }^{19}$. Para todos los que pertenecemos a otros ámbitos culturales y desde ellos investigamos la obra de Schutz, el legado de Lester Embree supondrá siempre una inspiración y un referente sólido. Este volumen contiene además cuatro textos de Schutz de 1956 editados por Embree relativos a unos discursos en el Institute on Ethics, que permiten al filósofo americano realizar un resumen de la posición ético-política de Schutz. Las discusiones durante los discursos versaron sobre problemas de igualdad de oportunidades en términos de diferentes barreras. Las intervenciones de Schutz se sitúan en el marco del texto "Equality and the Meaning Structure of the Social World" (1955) 20.

Como ya se indicó anteriormente, el último volumen que Embree dedicó exclusivamente al pensamiento de Schutz se centra en su teoría de las ciencias culturales: The Schutzian Theory of the Cultural Sciences (2015). Embree se

18 Lester Embree, "Disciplines beyond Philosophy: Recollecting a Phenomenological Frontier", en Lester Embree / Thomas Nenon (eds.), Phenomenology 2005, Vol. 5, Part 1: Selected Essays from North America, Bucharest, Zeta Books, 2007, p. 282.

${ }^{19}$ Cfr. ibid., pp. 271-282.

${ }^{20}$ Alfred Schutz, Collected Papers II: Studies in Social Theory, ed. A. Brodersen, The Hague, Martinus Nijhoff, 1964, pp. 226-273. 
lamenta de que la mayor parte de los investigadores en fenomenología no tienen en cuenta el proyecto de Husserl como una Wissenschaftslehre, traducido al inglés como "theory of science" o "science theory". Schutz sí estuvo interesado en esta disciplina a la cual se refirió en ocasiones como "methodology", en una época en la cual el término no estaba tan relacionado con el uso de técnicas estadísticas y lógicas en la ciencia ${ }^{21}$. Según Embree los científicos tienden a ocuparse de sus disciplinas en particular, mientras que los filósofos, como Schutz, se dedican al estudio de aspectos comunes a todas las especies y géneros dentro de la ciencia22. Nueve de los capítulos de The Schutzian Theory of the Cultural Sciences están dedicados al estudio de disciplinas particulares. Algunos de ellos estudian ciencias culturales a las cuales el propio Schutz se refirió, como Economía, Jurisprudencia, Ciencia Política, Sociología y Psicología. Además se dedican cuatro capítulos a disciplinas tratadas en el estilo de Schutz, aunque no estén estrictamente basadas en trabajos del filósofo austríaco. Se trata de Arqueología, Antropología Cultural, Enfermería y Psicoterapia. A excepción de la Arqueología en The Schutzian Theory of the Cultural Sciences no se incluyen ciencias históricas ya que, como Embree nos recuerda, Schutz dedicó a la Historia el parágrafo 42 de su obra de $1932^{23}$. Los últimos capítulos de The Schutzian Theory of the Cultural Sciences están dedicados al estudio de algunas cuestiones filosóficas del pensamiento de Schutz de las que se ocupó Embree durante su vida acerca de las cuales venía publicando a lo largo de estos últimos años. Destacan ensayos sobre metodología, tipos ideales, el concepto de significado, grupos sociales, relevancia o significatividad y verificabilidad. En definitiva esta última aportación de Embree al estudio de Alfred Schutz en forma de volumen monográfico nos presenta una sólida teoría de las ciencias culturales, junto con el análisis de algunas de ellas a partir de fragmentos de la diseminada obra de Schutz desde su época americana. Lester Embree va más allá de la letra del propio Schutz para realizar valiosas aportaciones en el espíritu del filósofo austríaco y nos deja muchos caminos abiertos de indudable interés, inspiradores para todo estudioso que se acerque a la obra de Alfred Schutz.

\footnotetext{
${ }^{21}$ Lester Embree, The Schutzian Theory of the Cultural Sciences, Dordrecht: Springer, 2015, p. VII.

22 Lester Embree, "Economics in the Context of Alfred Schutz's Theory of Science", Schutzian Research, vol. 1 (2009), p. 166. Publicado en castellano: Areté. Revista de Filosofía, vol. XVIII, 2 (2006), p. 310.

${ }^{23}$ Lester Embree, The Schutzian Theory of the Cultural Sciences, Dordrecht: Springer, 2015, p. VIII.
} 


\section{Repertorio Bibliográfico: Lester Embree sobre Alfred Schutz}

\subsection{Ediciones de libros y ensayos de Alfred Schutz}

"Letters of Schutz to Felix Kaufmann" (1930-1945), en Alfred Schutz, Collected Papers volumen V: Phenomenology and the Social Sciences, Dordrecht: Springer, 2011, pp. 209-213, procedente de Alfred Schutz Papers, General Manuscripts 129, Yale University, Beinecke Rare Book and Manuscript Library, General Collection of Rare Books and Manuscripts, Series 3, Box 27, Folder 631, tr. Michael Walter.

"Letters of Alfred Schutz with Aron Gurwitsch" (1939-1959), en Alfred Schutz, Collected Papers, Vol. V: Phenomenology and the Social Sciences, Dordrecht: Springer, 2011, pp. 227-273. Selección de cartas. Previamente publicadas como Alfred Schutz, Aron Gurwitsch: Briefwechsel, 1939-1959, München: Wilhelm Fink Verlag, 1985; version inglesa: Philosophers in Exile: The Correspondence of Alfred Schutz and Aron Gurwitsch, 1939-1959, ed. Richard Grathoff, trad. J. Claude Evans, Bloomington / Indianapolis: Indiana University Press, 1989.

"The Theory of Social Action: Texts and Letters with Talcott Parsons" (1940-41), en Alfred Schutz, Collected Papers, Vol. V: Phenomenology and the Social Sciences, Dordrecht: Springer, 2011, pp. 5-74. Selección de cartas. Previamente publicadas como The Theory of Social Action: The Correspondence of Alfred Schutz and Talcott Parsons, ed. Richard Grathoff, Bloomington / London: Indiana University Press, 1978.

"Letters of Schutz to Eric Voegelin" (1940-1952), en Alfred Schutz, Collected Papers, Vol. V: Phenomenology and the Social Sciences, Dordrecht: Springer, 2011, pp. 215-225. Selección de cartas. Previamente publicadas en Eine Freundschaft, die ein Leben ausgehalten hat: Briefwechsel (1938-1959), ed. Gerhard Wagner / Gilbert Weiss, trad. Michael Walter, Konstanz: UVK, 2004,. Publicadas posteriormente en $A$ Friendship that Lasted a Lifetime: The Correspondence Between Alfred Schütz and Eric Voegelin, trad. William Petropulos, Columbia / London: University of Missouri Press, 2011. 
"Choice and the Social Sciences" (1945), en Lester Embree (ed.), Life-World and Consciousness: Essays for Aron Gurwitsch, Evanston: Northwestern University Press, 1972, pp. 565-590; posteriormente publicado en Alfred Schutz, Collected Papers, Vol. V: Phenomenology and the Social Sciences, Dordrecht: Springer, 2011, pp. 75-92

"Outlines on 'Relevance and Action'" (1947), en Alfred Schutz, Collected Papers, Vol. V: Phenomenology and the Social Sciences, trad. Michael Walter, Dordrecht: Springer, 2011, pp. 201-208; previamente publicado como "Wiener Exzerpte", en Alfred Schutz Werkausgabe, ed. Richard Grathoff / Hans-Georg Soeffner / Ilja Srubar, en Vol. VI-1: Relevanz und Handeln I: Zur Phänomenologie des Alltagswissens, ed. Elisabeth List, Konstanz: UVK, 2004, pp. 45-54.

"Reflections on the Problem of Relevance" (1947, 1951), en Alfred Schutz, Collected Papers, Vol. V: Phenomenology and the Social Sciences, Dordrecht: Springer, 2011,pp. 93-199; previamente publicado como Reflections on the Problem of Relevance, ed. Richard Zaner, New Haven: Yale University Press, 1970.

"Sartre's Theory of Alter Ego" (1948), en Dermot Moran / Lester Embree (eds.), Phenomenology: Critical Concepts in Philosophy, Vol. 5, London: Routledge, 2004, pp. 309-327; previamente publicado en Alfred Schutz, Collected Papers, Vol. I: The Problem of Social Reality, ed.Maurice Natanson, The Hague: Martinus Nijhoff, 1962, pp. 180-203.

"Positivistic Philosophy and the Actual Approach of Interpretative Social Science" (1953), Husserl Studies, vol. 14 (1997) 125-149; posteriormente publicado en Dermot Moran / Lester Embree (eds.), Phenomenology: Critical Concepts in Philosophy, Vol. III, London: Routledge, 2004, pp. $119-145$

"T. S. Eliot's Theory of Culture" (1953), en Alfred Schutz, Collected Papers, Vol. V: Phenomenology and the Social Sciences, Dordrecht: Springer, 2011, pp. 275-289.

"Sociological Aspect of Literature" (1955), en Lester Embree (ed.), Alfred Schutz's "Sociological Aspect of Literature": Construction and 
Complementary Essays, Dordrecht: Kluwer Academic Publishers, 1998, pp. 4-7.

"Schutz's Contributions in the General Discussion at the Institute" (1956), en Lester Embree (ed.), Schutzian Social Science, Dordrecht: Kluwer Academic Publishers, 1999, pp. 244s, 247-249, 250-252, 253s, 258s, $264 s, 272-275$.

"Some Considerations concerning Thinking in Terms of Barriers" (1956), en Lester Embree (ed.), Schutzian Social Science, Dordrecht: Kluwer Academic Publishers, 1999, pp. 287-289.

"Memorandum" (1956), en Lester Embree (ed.), Schutzian Social Science, Dordrecht: Kluwer Academic Publishers, 1999, pp. 291-295.

"Report on the Discussions of Barrieres to Equality of Opportunity for the Development of Powers of Social and Civil Judgement" (1956), en Lester Embree (ed.), Schutzian Social Science, Dordrecht: Kluwer Academic Publishers, 1999, pp. 297-311.

"Letter of Alfred Schutz to Clarence H. Faust, the Fund for the Advancement of Education" (1957), en Lester Embree (ed.), Schutzian Social Science, Dordrecht: Kluwer Academic Publishers, 1999, pp. 313-318.

"The Problem of Transcendental Intersubjetivity in Husserl" (1957), Dermot Moran / Lester Embree (eds.), Phenomenology: Critical Concepts in Philosophy, Vol. II, London: Routledge, 2004, pp. 143-178; previamente publicado en Alfred Schutz, Collected Papers, Vol. III: Studies in Phenomenological Philosophy editado por Ilse Schutz, introducido por Aron Gurwitsch, The Hague: Martinus Nijhoff, 1970, pp. 51-91.

"The Meaning Structure of the Social World" (1958), en Lester Embree (ed.), Schutzian Social Science, Dordrecht: Kluwer Academic Publishers, 1999, pp. IX-X.

"Husserl and his Influence on me" (1958), ed. Lester Embree, The Annals of Phenomenological Sociology, vol. 2 (1977), pp. 40-44; posteriormente publicado en Alfred Schutz, Collected Papers, Vol. V: Phenomenology and the Social Sciences, Dordrecht: Springer, 2011, pp. 1-4. 
"Problems of a Sociology of Language" (Fall Semester, 1958), ed. Lester Embree / Fred Kersten, Schutzian Research, vol. 2 (2010) 63-107.

Collected Papers, Vol V: Phenomenology and the Social Sciences, ed. Lester Embree, Dordrecht: Springer, 2011.

\subsection{Libros de Lester Embree como autor, editor o co-editor}

Life-World and Consciousness: Essays for Aron Gurwitsch, ed. Lester Embree, Evanston: Northwestern University Press, 1972.

Worldly Phenomenology: The Continuing Influence of Alfred Schutz on North American Human Science, ed. Lester Embree, Washington, D.C.: Center for Advanced Research in Phenomenology / University Press of America, 1988.

Japanese and Western Phenomenology, eds. Philip Blosser / Eiichi Schimomissé / Lester Embree / Hiroshi Kojima, Dordrecht: Kluwer Academic Publishers, 1993.

Phenomenology of the Cultural Disciplines, eds. Daniel Mano / Lester Embree, Dordrecht: Kluwer Academic Publishers, 1994.

Encyclopedia of Phenomenology, ed. in chief Lester Embree, Dordrecht: Kluwer Academic Publishers, 1997.

The Phenomenology of Values and Valuing, eds. James G. Hart / Lester Embree, Dordrecht: Kluwer Academic Publishers, 1997 (contiene el artículo de Robert W. Jordan, "The Part Played by Value in the Modification of Open into Attractive Possibilities", pp. 81-94).

Alfred Schutz's "Sociological Aspect of Literature": Construction and Complementary Essays, ed. Lester Embree, Dordrecht: Kluwer Academic Publishers, 1998.

Schutzian Social Science, ed. Lester Embree, Dordrecht: Kluwer Academic Publishers, 1999.

Feminist Phenomenology, eds. Linda Fisher / Lester Embree, Dordrecht: Kluwer Academic Publishers, 2000 (contiene el artículo de Louise Levesque- 
Lopman, "Listen and You Will Hear: Reflections on Interviewing from a Feminist Phenomenological Perspective", pp. 103-132).

Phenomenology of the Political, eds. Kevin Thompson / Lester Embree, Dordrecht: Kluwer Academic Publishers, 2000.

The Reach of Reflection. Issues for Phenomenology's Second Century, Vol. 1-2, eds. Steven Crowell / Lester Embree / Samuel J. Julian, Boca Raton, FL: Center for Advanced Research in Phenomenology, 2001.

Phenomenological Approaches to Moral Philosophy, eds. John J. Drummond / Lester Embree, Dordrecht: Kluwer Academic Publishers, 2002 (contiene el artículo de Michael Barber, "Alfred Schutz: Reciprocity, Alterity, and Participative Citizenry", pp. 415-435).

Essays in Celebration of the Founding of the Organization of Phenomenological Organizations, eds. Chan-Fai Cheung / Ivan Chvatik / Ion Copoeru / Lester Embree / Julia Iribarne / Hans Rainer Sepp, en www.o-p-o.net, 2003.

Phenomenology: Critical Concepts in Philosophy, 5 Vols., eds. Dermont Moran / Lester Embree, London: Routledge, 2004.

Phenomenology 2005, Vol. 5, Part 1 \& 2: Selected Essays from North America, eds. Lester Embree / Thomas Nenon, Bucharest: Zeta Books, 2007 (contiene el artículo de Michael Barber, "Phenomenology and the Schutz/Voegelin Correspondence, pp. 37-66").

Environment, Technology, Justification: Reflective Analyses, Zeta Books, Bucharest: Zeta Books, 2008.

Alfred Schutz and his Intellectual Partners, eds. Hisashi Nasu / Lester Embree / George Psathas / Ilja Srubar, Konstanz: Universitaetsverlag Konstanz, 2009.

Handbook of Phenomenological Aesthetics, eds. Hans Rainer Sepp / Lester Embree, Dordrecht: Springer, 2010.

Husserl's Ideen, eds. Lester Embree / Thomas Nenon, Dordrecht: Springer, 2013 (contiene el artículo de Michael Barber, "Alfred Schutz and the Problem of Empathy", pp. 313-326). 
The Schutzian Theory of the Cultural Sciences, Dordrecht: Springer, 2015.

Phenomenology: Essays in Memory of Petee Jung, eds. Hwa Yol Jung / Lester Embree, Dordrecht: Springer, 2016.

The Golden Age of Phenomenology at the New School for Social Research, 19541973, eds. Lester Embree / Michael Barber, Ohio: Ohio University Press, 2017.

\subsection{Capítulos de Libros y Artículos de Revistas de Lester Embree}

"Everyday Social Relevancy in Gurwitsch and Schutz", Annals of Phenomenological Sociology vol. 2 (1977) 45-61.

"Methodology is where Human Scientists and Philosophers can meet: Reflections on the Schutz-Parsons Exchange", Human Studies, vol. 3 (1980) 367373.

"Review of Alfred Schutz, An Intellectual Biography, Helmut R. Wagner", International Studies in Philosophy, vol. 19, 3 (1987) 119-122.

"Introduction", en Worldly Phenomenology: The Continuing Influence of Alfred Schutz on North American Human Science, ed. Lester Embree, Washington, D.C.: Center for Advanced Research in Phenomenology / University Press of America, 1988, pp. XI-XVII.

"Schutz on Science", en Worldly Phenomenology: The Continuing Influence of Alfred Schutz on North American Human Science, ed. Lester Embree, Washington, D.C.: Center for Advanced Research in Phenomenology / University Press of America, 1988, pp. 251-274.

"Schütz's Phenomenology of the Practical World", en Ilja Srubar / Elizabeth List (eds.), Alfred Schütz, Neue Beiträge zur Rezeption seines Werkes, Amsterdam: Rodopi, 1988, pp. 121-144.

"Notes on the specification of 'meaning' in Schutz", Human Studies, vol. 14 (1991) 207-218.

"In Memoriam: Ilse Schutz (1902-1990)", Human Studies, vol. 14 (1991) 219224. "Introduction: Reflection on the Cultural Disciplines", en Daniel 
Mano / Lester Embree (eds.), Phenomenology of the Cultural Disciplines, Dordrecht: Kluwer Academic Publishers, 1994, pp. 1-37.

"Ethnic-Studies as Multi-Discipline and Phenomenology", coed. Stanford Lyman, en ibid., pp. 211-249.

"Editor's Preface" a "Positivistic Philosophy and the Actual Approach of Interpretative Social Science: An Ineditum of Alfred Schutz from Spring 1953", Husserl Studies, vol. 14, (1997) 123-125.

"Introduction", coed. J.N. Mohanty, en Encyclopedia of Phenomenology, Dordrecht: Kluwer Academic Publishers, 1997, pp. 1-10.

"Constitutive Phenomenology of the natural Attitude", en ibid., pp. 114-116.

"Cultural Disciplines", en ibid., pp. 121-123.

"Ethnic Studies", coed. Stanford Lyman, en ibid., pp. 194-198.

"Aron Gurwitsch", en ibid., pp. 284-287.

"Human Sciences", en ibid., pp. 315-321.

"Naturalism", en ibid., pp. 480-485.

"United States of America", coeds. J. Edie / D. Inde / J. Kockelmans / C. Schragen, en ibid., pp. 718-723.

"A Construction of Alfred Schutz's 'Sociological Aspect of Literature", en Lester Embree (ed.), Alfred Schutz's "Sociological Aspect of Literature": Construction and Complementary Essays, Dordrecht: Kluwer Academic Publishers, 1998, pp. 3-71.

"Preface" en Lester Embree (ed.), Schutzian Social Science, Dordrecht: Kluwer Academic Publishers, 1998, pp. XI-XIV.

"The Ethical-Political Side of Schutz: his contributions at the 1956 Institute on Ethics concerned with Barriers to Equality of Opportunity", en Lester Embree (ed), Schutzian Social Science, Dordrecht: Kluwer Academic Publishers, 1998, pp. 235-285.

"Los grupos en Schutz: la estructura significativa concreta del mundo sociohistórico", Franciscanum: Revista de las Ciencias del Espíritu, vol. 41, 
122-123 (1999)187-194; posteriormente en versión original como "Groups in Schutz: The Concrete Meaning Structure of the SocioHistorical World", PhaenEx: Journal of Existential and Phenomenological Theory and Culture, vol. 6, 1 (2011) 1-11.

"A Problem in Alfred Schutz's Methodology of the Cultural Sciences" - revisado y ampliado-, en Hisashi Nasu / Frances Ch. Waksler (eds.), Interaction and Everyday Life: Phenomenological and Ethnomethodological Essays in Honor of George Psathas, Lanham: Lexington Books, 2012, pp. 89102; previamente se publicó en japonés en Bunku to Shakai, vol. 1 (1999) pp. 105-31

"O Atractivo de Alfred Schutz en disciplinas fora da Filosofía, como a Xurisprudencia", Agora. Papeles de Filosofía, vol. 19, 1 (2000), pp. 1530; posteriormente publicado en versión original como "The Appeal of Alfred Schutz beyond Philosophy, e.g. Jurisprudence", en Martin Endress / George Psathas / Hisashi Nasu (eds.), Explorations of the Life-World: Continuing Dialogues with Alfred Schutz, Dordrecht: Springer, 2005, pp. 77-95.

"Alfred Schutz on Reducing Social Tensions", en Phenomenology of the Political, eds. Kevin Thompson / Lester Embree, Dordrecht: Kluwer Academic Publishers, 2000, pp. 81-102.

"Phenomenology of Nursing as a Cultural Discipline", coautora Florence Tocantins, en The Reach of Reflection. Issues for Phenomenology's Second Century, Vol. 2, eds. Steven Crowell / Lester Embree / Samuel J. Julian, Boca Raton, FL: Center for Advanced Research in Phenomenology, 2001, pp. 364-383.

"Alfred Schutz", The Internet Encyclopedia of Philosophy (2002), online: http://www.iep.utm/schutz - recuperado en 09/2017.

"Husserl as a Trunk of the American Continental Tree", International Journal of Philosophical Studies, vol. 11, 2 (2003) 177-190.

"Reflective Analysis in and of Social Psychology: A Model for Interdisciplinary Phenomenology", en Essays in Celebration of the Founding of the Organization of Phenomenological Organizations, eds. Chan-Fai Cheung 
/ Ivan Chvatik / Ion Copoeru / Lester Embree / Julia Iribarne / Hans Rainer Sepp, en www.o-p-o.net, 2003.

"The Possibility of a Constitutive Phenomenology of the Environment", en Charles S. Brown / Ted Toadvine (eds.), Eco-Phenomenology, Albany, NY: Suny Press, 2003, pp. 37-50, posteriormente publicado en MORAN, Dermot y EMBREE, Lester (Eds.) (2004) vol. IV, pp. 136-148.

"A Problem in Schutz's Theory of the Historical Sciences with an Ilustration from the Women's Liberation Movement", Human Studies, vol. 27 (2004) 281306.

"Phenomenology of the Consocial Situation: Advancing the problems", en David Carr / Chan-Fai Cheung (eds.), Space, Time and Culture, Dordrecht: Kluwer Academic Publishers, 2004, pp. 119-133.

"Alfred Schutz", en John Shook (ed.), Dictionary of Modern American Philosophers, Bristol: Thoemmes Continuum, 2000.

"La economía en el contexto de la teoría de la ciencia de Alfred Schutz", Areté. Revista de Filosofía, vol. XVIII, 2 (2006) 309-322; posteriormente publicado en versión original como "Economics in the Context of Alfred Schutz's Theory of Science", Schutzian Research, vol. 1 (2009) 165-175.

"Introduction to Volume V: Phenomenology across Disciplines in North America at the Begining of the 21th Century", coed. Thomas Nenon, Phenomenology 2005, Vol. 5, Part 1 \& 2: Selected Essays from North America, eds. Lester Embree / Thomas Nenon, Bucharest: Zeta Books, 2007, pp. 13-24.

"Disciplines Beyond Philosophy: Recollecting a Phenomenological Frontier", en ibid., pp. 271-282.

"The Constitution of Basic Culture", en Environment, Technology, Justification: Reflective Analyses, Zeta Books, Bucharest: Zeta Books, 2008, pp. 87101; publicado en castellano como "La constitución de la cultura básica", en César Moreno / Alicia de Mingo (eds.) (2005), Signo, intencionalidad, verdad. Estudios de fenomenología, Sevilla: Sociedad Española de Fenomenología / Universidad de Sevilla, pp. 345-355. 
"A Beginning for the Phenomenological Theory of Primate Ethology", Environmental Philosophy, vol. 5, 1 (2008) 61-74.

"The Nature and Role of Phenomenological Psychology in Alfred Schutz", Journal of Phenomenological Psychology, vol. 39 (2008) 141-150.

"Book Symposium: Schutz, Seebohm, and Cultural Science", International Journal of Philosophical Studies, vol. 16, 5 (2008) 731-744.

"Dorion Cairns, Alfred Schutz, and the Egological Reduction" en Alfred Schutz and his Intellectual Partners, eds. Hisashi Nasu / Lester Embree / George Psathas / Ilja Srubar, Konstanz: Universitaetsverlag Konstanz, 2009, pp. 177-216.

"Some Philosophical Differences Within a Friendship: Gurwitsch and Schutz", en ibid., pp. 231-254.

"Phenomenology and Social Constructionism: Constructs for Political Identity", Journal of Phenomenological Psychology, vol. 40, 2 (2009) 127-139.

"Intra-culturalidad: género, generación y relaciones de clase en Schutz", en Acta fenomenológica latinoamericana, Vol. III, Morelia: Universidad Michoacana de San Nicolás de Hidalgo, 2009, pp. 179-193.

"Introduction", coed. Hans Rainer Sepp, en Handbook of Phenomenological Aesthetics, eds. Hans Rainer Sepp / Lester Embree, Dordrecht: Springer, 2010, pp. XV-XXX.

"Methodology", en ibid., pp. 215-221.

"Preface" y "Introduction", en "Alfred Schutz, Problems of a Sociology of Language (Fall Semester, 1958)", Schutzian Research, vol. 2 (2010) 5560.

"Founding Some Practical Disciplines in Schutzian Social Psychology", Bulletin d'Analyse Phénoménologique, vol. VI, 1 (2010) 1-11.

"Cultural Anthropology in Schutzian Perspective", Phainomena: Journal of the Phenomenological Society of Ljubljana, vol. 19, 74-75 (2010) 217-228.

"Interdisciplinarity within Phenomenology", Indo-Pacific Journal of Phenomenology, vol. 10, 1 (2010) 1-7; publicado en castellano como "La 
interdisciplinariedad dentro de la fenomenología", trad. Luis R. Rabanaque, Investigaciones Fenomenológicas 8 (2011) 9-21.

"Introduction", en Alfred Schutz Collected Papers, Vol V: Phenomenology and the Social Sciences, ed. Lester Embree, Dordrecht: Springer, 2011, pp. XI$\mathrm{XV}$.

"Phenomenological Nursing in Schutzian Perspective", en Osborne Wiggins / P. Allen / Annette C Allen (eds.), Clinical Ethics and the Necessity for Stories: Essays in Honor of Richard M. Zaner, Dordrecht: Springer, 2011, pp. 87-97.

"Del 'Nosaltres' al 'Jo' I de tornado: Aprenent encara de la New School", Anuari de la Societat Catalana de Filosofia, vol. XXII (2011) 53-62.

"A Problem in Alfred Schutz's Methodology of the Cultural Sciences", en Hisashi Nasu / Frances Ch. Waksler (eds.), Interaction and Everyday Life: Phenomenological and Ethnomethodological Essays in honor of George Psathas, Lanham: Lexington Books, 2012, pp. 89-102 -versión revisada y ampliada de texto traducido al japonés en Bunku to Shakai, vol. 1 (1999) 105-31.

"It's about Time! A Sometimes Personal Narrative of Schutz Scholarship", Schutzian Research, vol. 4 (2012) 9-22.

"Two Concepts of Type in the Work of Alfred Schutz", Schutzian Research, vol. 4 (2012) 125-131.

"Welcome to the phenomenological tradition!", South African Journal of Philosophy, vol. 32, 4 (2013) 395-399.

"Cultural Science in Literary Light", en Michael Barber / Jochen Dreher (eds.), The Interrelation of Phenomenology, Social Sciences and the Arts, Dordrecht: Springer, 2014, pp. 21-30.

"The Interpretationism of Alfred Schutz or How Woodcutting Can Have Referential and Non-Referential Meaning", en Michael Staudigl / George Berguno (eds.), Schutzian Phenomenology and Hermeneutic Traditions, Dordrecht: Springer, 2014, pp. 69-79. 
"Constructing a Schutzian Theory of Political Science", en Phenomenology: Essays in Memory of Petee Jung, en Hwa Yol Jung / Lester Embree (eds.), Dordrecht: Springer, 2016, pp. 59-75. 